\title{
The polony phantom: a cost-effective aid for teaching emergency ultrasound procedures
}

\author{
Mike Wells • Lara Goldstein
}

Received: 3 December 2009 /Accepted: 27 December 2009 / Published online: 30 March 2010

(C) Springer-Verlag London Ltd 2010

\begin{abstract}
Background Central venous access and nerve blockade are essential skills in the practice of emergency medicine, and the utility of emergency ultrasound to guide these procedures has been well demonstrated.

Methods A phantom is a valuable tool to learn and develop the dexterity for ultrasound-guided techniques.

Results To date, the time and cost required to produce or purchase such models has somewhat limited their use.

Conclusion A time- and cost-effective alternative using polony and common household items is presented.
\end{abstract}

Keywords Imaging phantoms · Ultrasonography · central venous catheterisation · Nerve block · Emergency ultrasound

\section{Introduction}

The use of emergency ultrasound (EUS) to assist with vascular access techniques is advocated by many organ-

M. Wells $\cdot$ L. Goldstein

Division of Emergency Medicine,

Faculty of Health Sciences,

University of the Witwatersrand,

7 York Road, Parktown,

Johannesburg, South Africa

M. Wells $(\square)$

Postnet suite 429 ,

Private Bag X1510,

Glenvista 2058, South Africa

e-mail: mike@casualty.co.za isations across the world including the American College of Emergency Physicians, the College of Emergency Medicine in the UK, the Australasian College of Emergency Medicine and the College of Emergency Medicine of South Africa. EUS has been shown to increase the success rate and decrease the complication rate of central venous cannulation [1] as well as plexus and peripheral nerve blockade. [2] These techniques should therefore be included in the instruction of basic and intermediate EUS. A phantom is generally used for the practical component of this teaching to encourage the development of appropriate hand-probe-eye co-ordination, but commercial phantoms are expensive and previously described home-made phantoms require time, skill and the availability of the necessary materials to assemble. [3-6]

We have developed a simple vascular access/nerve block phantom made from a polony (bologna) roll that can be constructed within 5 minutes with minimal skill and without the need for other materials.

\section{Methods}

Polony is a highly processed mixed-meat meat-roll that is encased in a thin plastic sausage-shaped covering. A 500-g roll of polony made from very finely minced meat was the best product that we found. Some polony products (especially pure poultry and pork-free polony) were not ground finely enough and, as a result, were too echogenic. We drilled a hole through the plastic casing of the polony at one end (see Fig. 1) with a 12-mm wood drill bit attached 


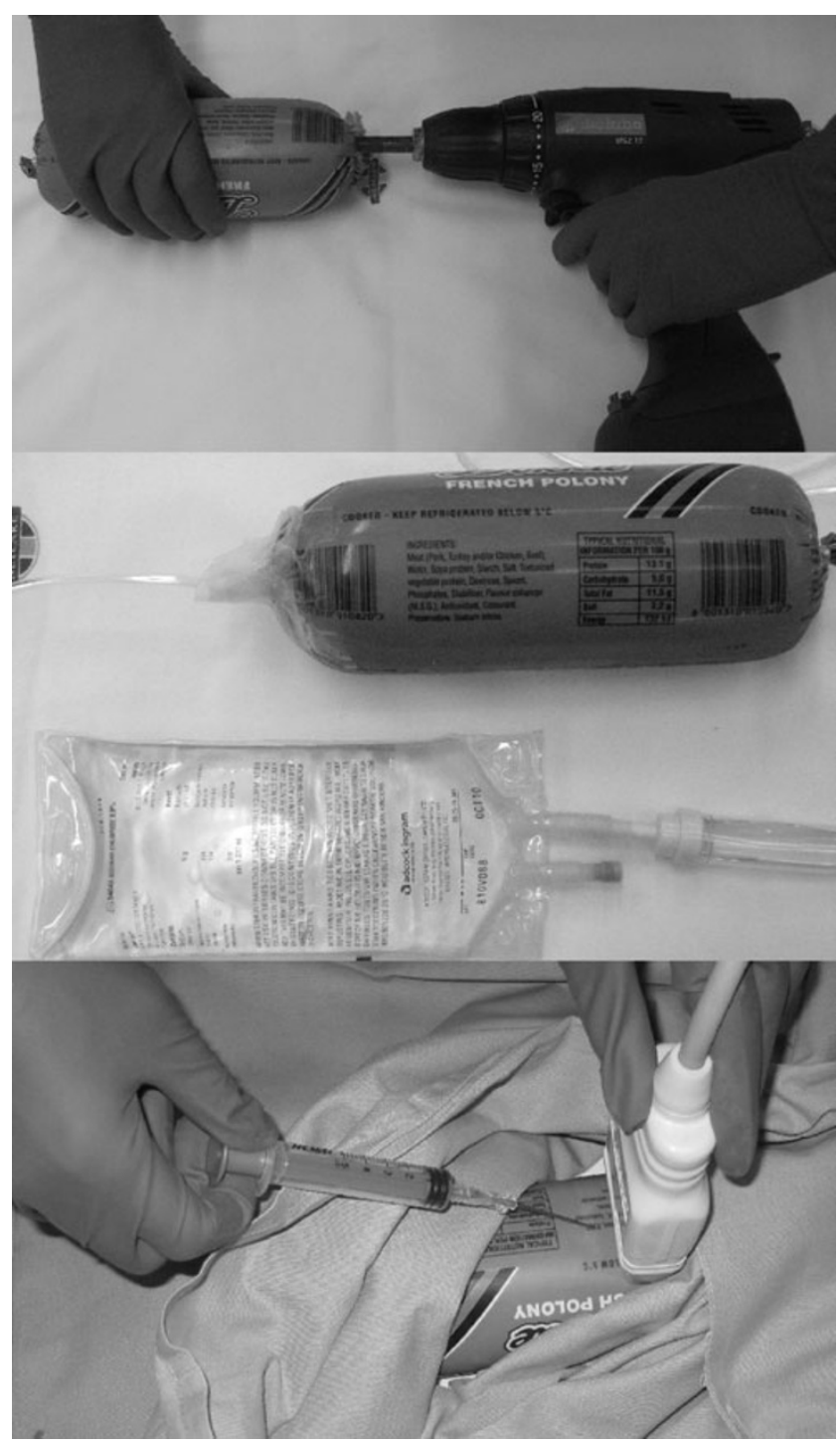

Fig. 1 The construction of the central venous access polony phantom. First a hole is carefully bored from one end of the polony with a lowspeed drill, then an expired IV is connected to keep the vein filled. Standard techniques can be used for the simulated vascular access

to a variable-speed, battery-powered hand drill. The drill bit was carefully advanced into the polony at a low speed with frequent withdrawal to remove debris from the drill bit. We thus created a long tunnel in the polony about $20 \mathrm{~mm}$ from the surface of the casing. We washed the debris out of the tunnel and then filled it with water, taking care to avoid air bubbles. We inserted the end of an intravenous (IV) infusion set (connected to a bag of IV saline into which a red dye was added) to the end of the tunnel and sealed it with tape applied circumferentially around the end of the polony. The phantom was then strapped to a kitchen cutting board and, once draped, was ready for use. When the phantom needed to be prepared some time before use, a cork was taped into the open end of the drilled hole once it had been washed out and filled with water. The polony phantom could be used with the cork still in place as an alternative to connecting an IV set.

To create the nerve phantom, we inserted a thin wire from one end of the polony to the other. We then attached a $600-\mathrm{mm}$ woven nylon shoelace to the end of the wire and pulled the lace back through the polony. This was done with the polony submerged underwater to prevent air entry into the tract. Tape was put on each end of the shoelace tract in order to create a watertight seal.

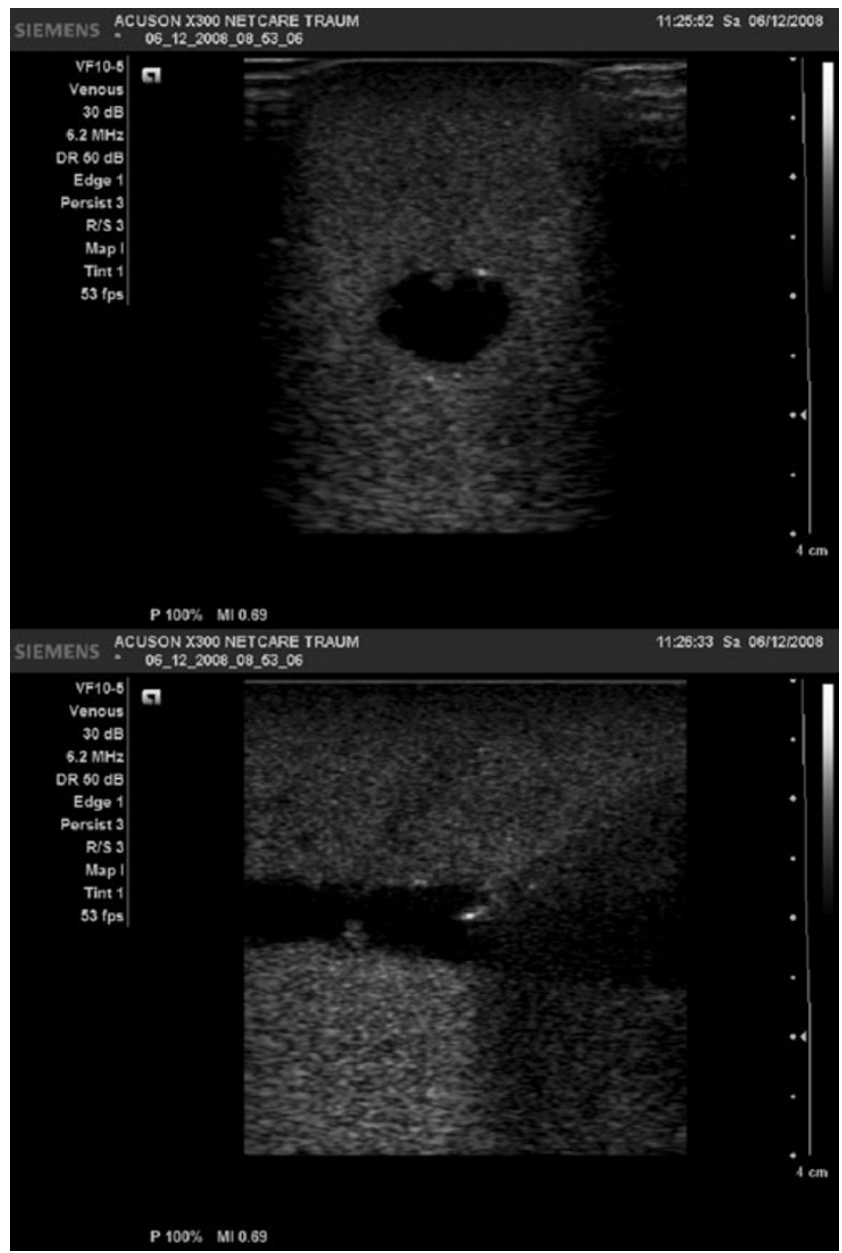

Fig. 2 Top: Image of the "vein" in transverse view with the tip of the needle visible in the channel. Bottom: The "vein" in longitudinal view with the needle visible in the lumen, which has created a prominent acoustic shadow (Siemens Acuson X300) 


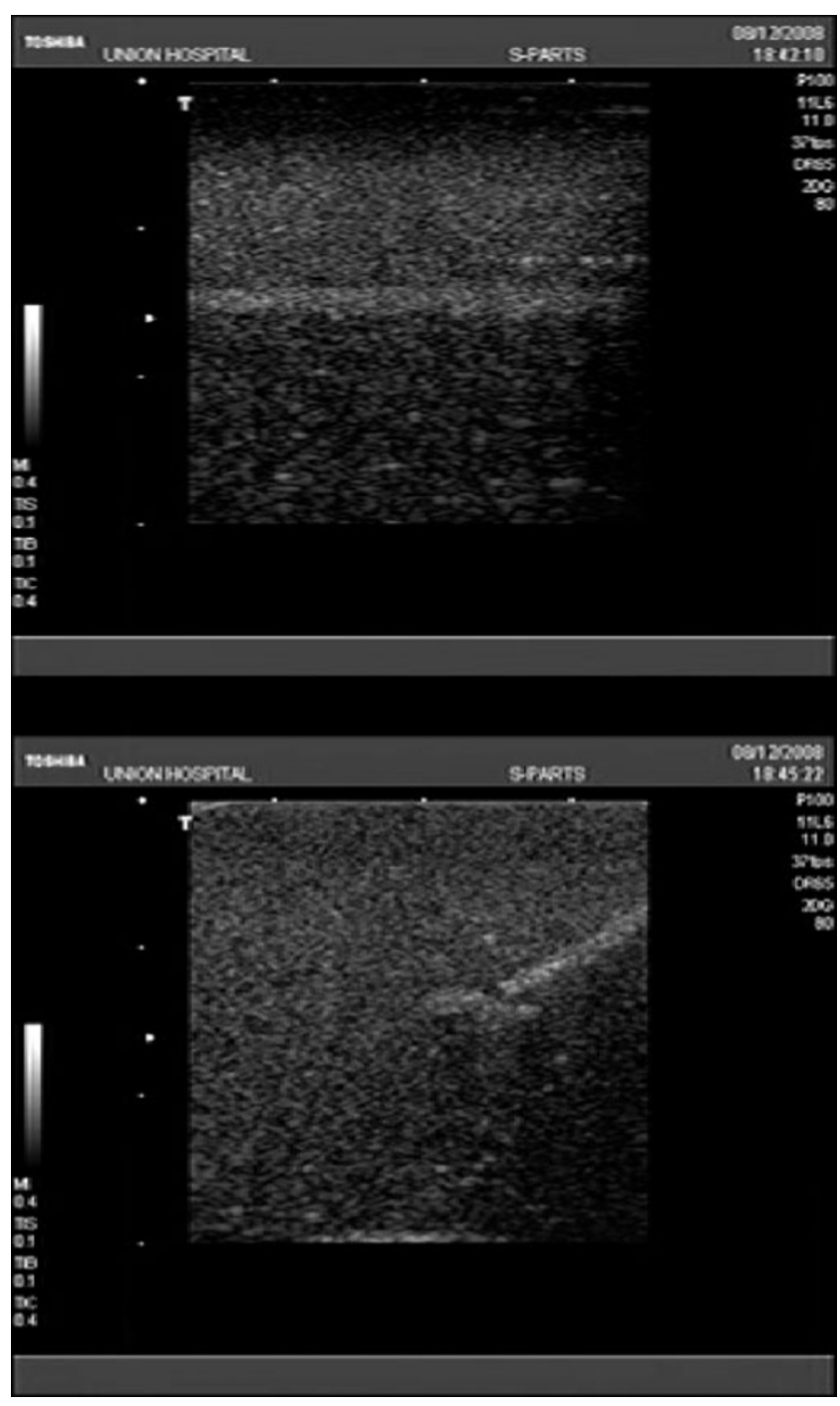

Fig. 3 Top: Image of the "nerve" in longitudinal view. Bottom: Image of the "nerve" in transverse view with the blocking needle approaching from the upper right of the image. The lucent area surrounding the "nerve" is the fluid that has been injected

\section{Results}

The polony phantom worked extremely well generating suitable ultrasound images for teaching the procedural techniques. The "vein" could be well demonstrated in both transverse and longitudinal orientations. The advancing needle could be detected from local tissue movement, direct visualisation of the needle itself and by detection of the acoustic shadow created by the needle (see Fig. 2). Both inplane and out-of-plane cannulation techniques could be practised. Penetration of the "vein" was confirmed by the withdrawal of fluid, which was replenished by the IV saline.

Similarly, the shoelace in the polony created an ultrasound picture comparable to that of an echogenic nerve. Nerve block techniques could be practised using both in-plane and out-of-plane approaches with this phantom (see Fig. 3).

Figure 4 shows the ultrasound images obtained from a regular pork polony, a processed "mini-ham" and a chicken polony. The quality of the ultrasound images varied from product to product. Some products were so echogenic that the "vein" could not be visualised at all.

\section{Discussion}

In order to give a "hands-on" feel when learning to cannulate vessels and perform nerve blocks via ultrasound guidance, various alternatives have been suggested to maintain the realism of the training model. Existing designs for training aids have varied from gelatine-based recipes for phantoms (home-made and commercially available) to the use of a number of different animal tissues. The dexterity required to juggle the ultrasound probe whilst performing procedures needs to be taught and practised prior to its application on a real patient. The ideal phantom should be inexpensive, simple to construct, not be time-consuming to produce and have a sonographic appearance similar to human tissue.

Most recipes for home-made phantoms are gelatinebased and require several hours of preparation time prior to use. Although animal tissue phantoms can allow for the practical feel of human tissue, they are expensive and very time-consuming to construct. Commercially produced phantoms are generally expensive in comparison to the cost of the polony phantom (the equivalent of US\$2 in South Africa).

Since not all polonies produce the same quality of ultrasound images, it may be necessary to experiment with several products until an ultrasound-friendly brand is identified.

The polony phantom is a solution to the problem of lengthy preparation times or the expense of other types of phantoms, while still fulfilling the other requirements of an acceptable ultrasound teaching aid.

\section{Competing Interests None}


a
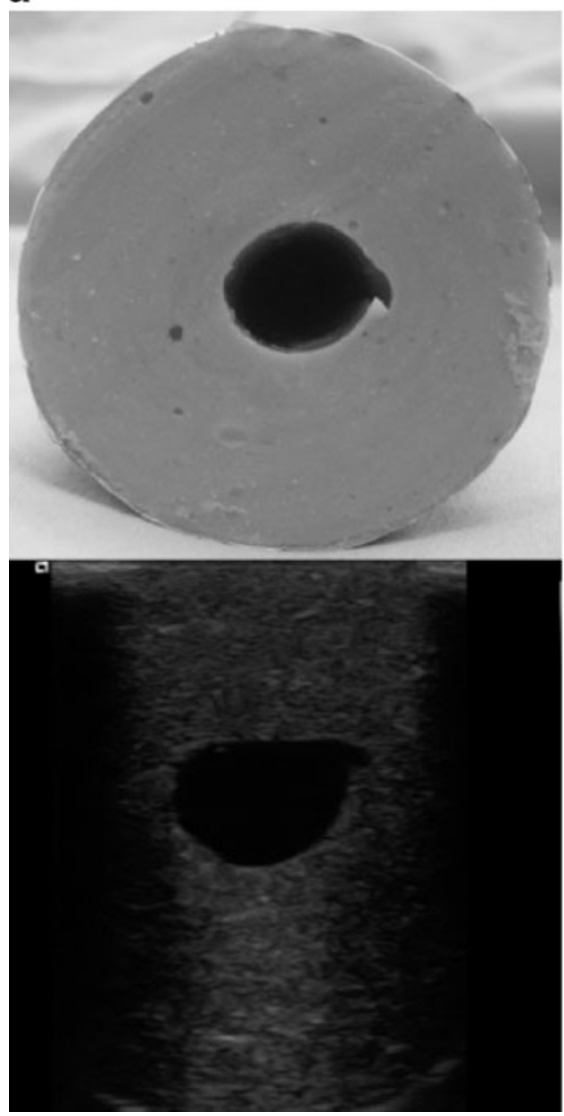

Fig. 4 a The ultrasound image generated from a regular pork polony. This is an excellent product to create a phantom for training. $\mathbf{b}$ The ultrasound image generated from a processed mini-ham that contains larger pieces of muscle tissue. The image is poorer than that of a more

\section{b}

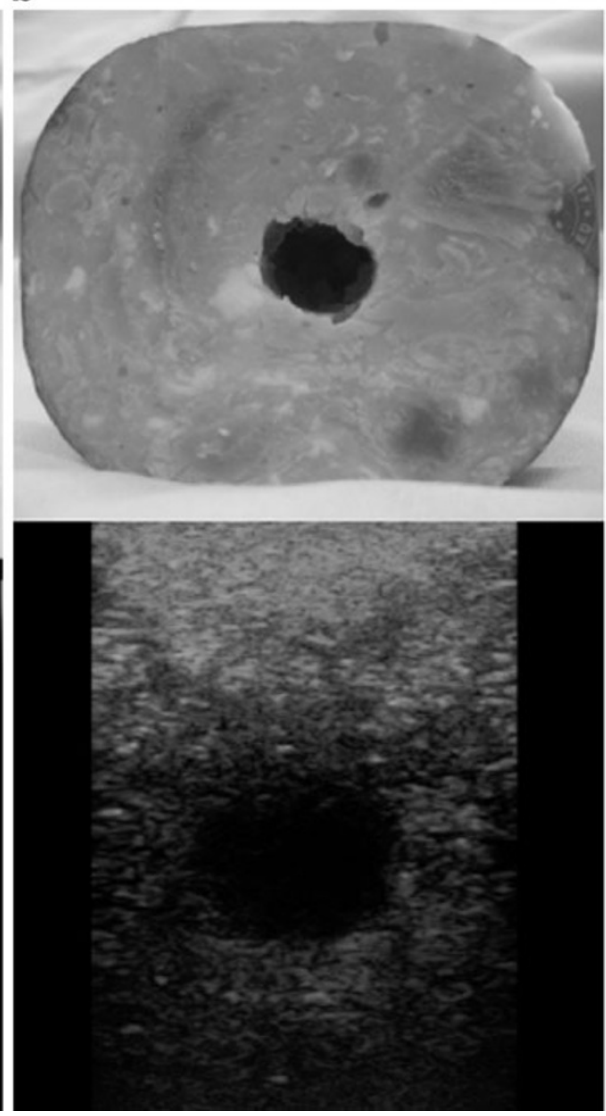

C

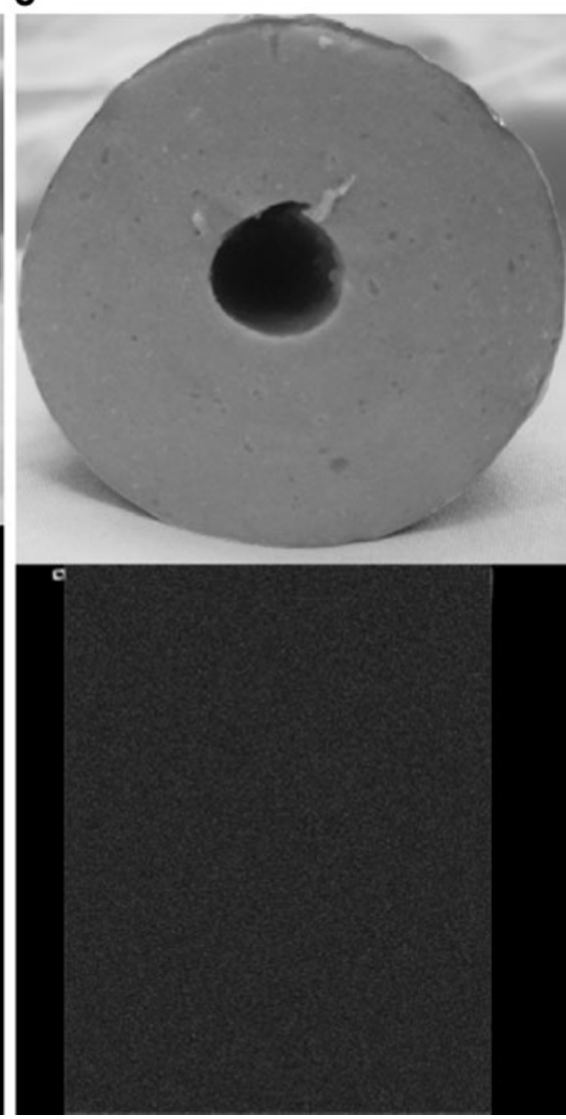

highly refined product. c The ultrasound image generated from a chicken polony. The images are poor because the meat is less finely ground and less homogenous. Some products, such as this one, are not useful for creating phantoms

\section{References}

1. Leung J, Duffy M, Finckh A (2006) Real-time ultrasonographicallyguided internal jugular vein catheterization in the emergency department increases success rates and reduces complications: a randomized, prospective study. Ann Emerg Med 48:540-547

2. Williams R, Saha B (2006) Best evidence topic report. Ultrasound placement of needle in three-in-one nerve block. Emerg Med J 23 (5):401-403

3. Kendall JL, Faragher JP (2007) Ultrasound-guided central venous access: a homemade phantom for simulation. Can J Emerg Med 9 (5):371-373

4. Osmer CL (2008) A gelatine-based ultrasound phantom. Anaesthesia 63:107

5. Xu D, Abbas S, Chan VWS (2005) Ultrasound phantom for handson practice. Reg Anesth Pain Med 30(6):593

6. Rose A, Reynolds F (2009) Ultrasound venous access simulation: the Italian job. Emerg Med J 26:76
Dr Wells is a specialist and consultant in Emergency Medicine with the Division of Emergency Medicine at the University of the Witwatersrand in Johannesburg, South Africa. He is the Director of Emergency Ultrasound training at the University and is involved in training in level 1 and level 2 ultrasound across the country. He is the Chairman of the Emergency Ultrasound Committee of the College of Emergency Medicine of South Africa. His research interests include emergency echocardiography, emergency ultrasound and paediatric emergency medicine, and he has a book in press on the use of ultrasound in regional anaesthesia techniques in the Emergency Department.

Dr Goldstein is a specialist and consultant in Emergency Medicine with the Division of Emergency Medicine at the University of the Witwatersrand in Johannesburg, South Africa. She is the resident programme director for the University. She is also involved in emergency ultrasound training in South Africa as well as the development and promotion of the use of ultrasound in prehospital medical care. 